\title{
Lunar Occultation of MACHOs
}

\section{Cheongho Han}

\section{Vijay K. Narayanan}

\section{Andrew Gould ${ }^{1}$}

\author{
Dept. of Astronomy, The Ohio State University, Columbus, OH 43210 \\ e-mail cheongho@payne.mps.ohio-state.edu \\ e-mail vijay@payne.mps.ohio-state.edu \\ e-mail gould@payne.mps.ohio-state.edu
}

\begin{abstract}
Lunar occultation can be used to measure the proper motions of some of the long time scale microlensing events, $t_{e} \gtrsim 70$ days, now being detected toward the Galactic bulge. The long events are difficult to explain within the context of standard models of the mass distribution and dynamics of the Galaxy. Han \& Gould (1995b) have suggested that they may be due to a kinematically cold population near the Sun. To resolve the mass, distance, and velocity of individual events and so to determine their nature, one must measure parallaxes and proper motions. For long events, parallaxes can be often obtained from ground-based measurements, but proper motions can only rarely be determined using conventional methods. Lunar occultations are therefore key to the understanding of the long events. We carry out realistic simulations to estimate the uncertainty of these measurements and show that proper motions could be measured for about one long event per year.
\end{abstract}

$\star^{1}$ Alfred P. Sloan Foundation Fellow 


\section{Introduction}

The MACHO (Alcock et al. 1995; Bennett et al. 1995) and OGLE (Udalski et al. 1994) collaborations have reported 54 candidate lensing events toward the Galactic bulge caused by Massive Compact Objects (MACHOs). The current estimate of the optical depth obtained by both groups is significantly higher than the theoretical estimate (Griest et al. 1991). Various solutions have been proposed to explain the observed optical depth excess. One solution assumes a bar-shaped Galactic bulge (Kiraga \& Pacyński 1994; Zhao, Spergel, \& Rich 1994). However, the excess optical depth is not completely solved even with the adoption of a triaxial bulge (Han \& Gould 1995a).

Recently, Han \& Gould (1995b) argued that the long events, those with time scales of $t_{e} \gtrsim 70$ days, cannot be explained within the context of the standard models of the mass distribution and stellar dynamics of the Galaxy, leaving the origin of these events as a puzzle. Although only three of the reported 54 events observed belong to this group, these long events contribute a significant fraction to the optical depth, provided that they are truly caused by MACHOs. Hence, their potential importance far exceeds their number. Han \& Gould (1995b) proposed that long events might be caused by a dynamically cold population of dark objects such as black holes or neutron stars. Being dynamically cold, such objects would be located very close to the Galactic plane, and thus only objects near the Sun could contribute to the events detected toward Baade's window located at $b=-3^{\circ} .9$. The objects would then have low transverse speeds and so long $t_{e}$.

The time scale, $t_{e}$, is the only observable from current observations, but from 
$t_{e}$ alone it is difficult to constrain the physical parameters of the long $t_{e}$ events. The time scale is related to the physical parameters by

$$
t_{e}=\frac{r_{e}}{v} ; \quad r_{e}^{2}=\frac{4 G M}{c^{2}} \frac{D_{\mathrm{ol}} D_{\mathrm{ls}}}{D_{\mathrm{os}}}
$$

Since there are three parameters, and only one measured quantity, the individual events are highly degenerate. Here $r_{e}$ is the Einstein ring radius, $M$ is the MACHO mass, $v$ is its transverse speed relative to the observer-source line of sight, and $D_{\mathrm{ol}}$, $D_{\mathrm{os}}$, and $D_{\mathrm{ls}}$ are the distances between the observer, lens, and source.

There have been two general approaches to break the degeneracy of the physical parameters; parallax and proper motion measurements. If the parallax is measured one can obtain the projected speed $\tilde{v}$ :

$$
\tilde{v}=\frac{D_{\mathrm{ol}}}{D_{\mathrm{ls}}} v
$$

The proper motion, $\mu$, is determined by measuring the angular size of the Einstein ring, $\theta_{e}$ :

$$
\mu=\frac{\theta_{e}}{t_{e}} ; \quad \theta_{e} \equiv \frac{r_{e}}{D_{\mathrm{ol}}}
$$

If both the proper motion and parallax of a MACHO are measured, one can obtain the distance to the MACHO by

$$
D_{\mathrm{ol}}=D_{\mathrm{os}}\left(\frac{\mu}{\tilde{v}} D_{\mathrm{os}}+1\right)^{-1}
$$

since the distance to the source stars $D_{\mathrm{Os}}=8 \mathrm{kpc}$ is known. Similarily $M=$ $\left(c^{2} / 4 G\right) t_{e}^{2} \tilde{v} \mu$ and $v^{-1}=\tilde{v}^{-1}+\left(\mu D_{\mathrm{os}}\right)^{-1}$. Therefore, the physical parameters of 
MACHOs would be uniquely determined from the combined information of parallax and proper motion.

For typical short events seen toward the bulge, parallax can be measured only from space (Gould 1994, 1995), and proper motions can be measured for only a few percent of events where the MACHO transits the face of the star (Gould 1994; Nemiroff \& Wickramasinghe 1994; Witt 1995). However, parallax measurements for long-time-scale events become feasible from the ground while proper motion measurement with traditional techniques becomes essentially impossible. Ground based parallax measurements are possible because the Earth moves through a substantial fraction of the Einstein ring during the event. Indeed Bennett et al. (1995) measured a parallax for one of the three long events using only the routine monitoring data (i.e., no followup photometry). On the other hand, proper motions from transits are rare because $\theta_{e} \gtrsim 1$ mas while the angular source size $\theta_{\star} \lesssim 10 \mu$ as, even for giants. However, large $\theta_{e}$ opens a possibility of resolving the two images of a lensed star and measuring their angular separation, $\phi_{\text {sep }}$. Once $\phi_{\text {sep }}$ is measured, one can uniquely determine $\mu$ (see $\S 2$ ). Due to the large $\theta_{e}$ of long events, it may one day be possible to measure $\phi_{\text {sep }}$ with high-resolution interferometry. Unfortunately, current interferometry has not achieved high enough resolution to resolve the expected very small separations of images; $\phi_{\text {sep }} \sim 3$ mas for long events (see $\S 3.2)$. Instead of interferometry, one can measure $\phi_{\text {sep }}$ by using lunar occultation. We show in $\S 5.2$ one can only measure the angular separation along a certain direction. However, this uncertainty can often be resolved with information about the lens geometry provided by parallax measurements. Even when the information from parallax measurement is inadequate, two lunar occultation measurements 
can resolve the degeneracy. Hence, lunar occultation gives enough information to determine the proper motion and so $M, v$, and $D_{\mathrm{ol}}$.

In this paper we analyze the possibility of measuring the angular separation of images of a gravitationally-lensed star using the lunar occultation method and determine the uncertainty of the measurement by carrying out realistic simulations. We find that if the observation is carried out for bright, moderate-highly magnified Galactic bulge giant stars, one can measure $\phi_{\text {sep }}$ with an uncertainty $\Delta \phi_{\text {sep }} \lesssim 1$ mas. The observational strategy and the method to find the intrinsic angular separation from the measured separation normal to the Moon's surface are discussed in $\S 5$.

\section{Proper Motion From Angular Separation Measurement}

The angular Einstein ring radius and therefore the proper motion of the long events can be measured if the angular separation of the two images of a lensed star is measured. The locations of the images of the star are the solution to the quadratic lens equation given by

$$
\theta_{\mathrm{I}}-\theta_{\mathrm{S}} \theta_{\mathrm{I}}-\theta_{e}^{2}=0
$$

where $\theta_{\mathrm{I}}$ and $\theta_{\mathrm{S}}$ are the image and source angle measured from the lens location. Then the angular separation of the two images is given by

$$
\phi_{\text {sep }}=\left|\theta_{I+}-\theta_{I-}\right|=\left(x^{2}+4\right)^{1 / 2} \theta_{e}
$$

where the dimensionless parameter $x$ is the separation between the source and lens measured in units of $\theta_{e}: x \equiv \theta_{\mathrm{S}} / \theta_{e}$. The value of $x$ is uniquely determined 
from the light curve because the magnification of the lensed star is related to $x$ by $A(x)=\left(x^{2}+2\right) / x\left(x^{2}+4\right)^{1 / 2}$. Thus, once the angular separation between two images of the source star is determined, one can obtain $\theta_{e}$.

\section{Diffraction Pattern by Lunar Occultation}

\subsection{Fresnel Diffraction}

A stellar image produces a Fresnel diffraction pattern when it is occulted by the Moon. In this case, the star is idealized as a point source and the Moon's disk as a semi-infinite plane. When images of a lensed star are blocked by the Moon, the observed diffraction pattern will differ slightly from a perfect pointsource pattern because the image of the lensed star is composed of two images with a small separation. By carefully analyzing the occultation of a lensed star, one can use this difference to measure the angular separation which cannot be resolved using current telescope technology. Fortunately, the Moon passes through the Galactic bulge every month and this permits one to apply the lunar occultation method to measure the separation of two images of a star lensed by a significant fraction of MACHOs. In addition, since very long events are suspected to be caused by MACHOs close to the Sun, the angular separations would be large enough to measure $\phi_{\text {sep }}$ precisely by the lunar occultation method.

The diffraction pattern produced by a background source star when it is occulted by an opaque object (e.g., the Moon) is given by

$$
\frac{g}{g_{0}}=\frac{1}{2}\left\{\left[\frac{1}{2}-\mathcal{C}(z)\right]^{2}+\left[\frac{1}{2}-\mathcal{S}(z)\right]^{2}\right\},
$$

where $\mathcal{C}$ and $\mathcal{S}$ are the Fresnel cosine and sine integrals and $g_{0}$ is the intensity 
without diffraction (e.g., Hecht \& Zajac 1979). The two Fresnel integrals are defined by

$$
\mathcal{C}(z)=\int_{0}^{z} \cos \left(\frac{\pi}{2} z^{\prime 2}\right) d z^{\prime}, \quad \mathcal{S}(z)=\int_{0}^{z} \sin \left(\frac{\pi}{2} z^{\prime 2}\right) d z^{\prime}
$$

Here the dimensionless distance variable $z$ is defined by

$$
z=r\left[\frac{2\left(d_{\mathrm{OM}}+d_{\mathrm{M}}\right)}{\lambda d_{\mathrm{OM}} d_{\mathrm{M}}}\right]^{1 / 2}
$$

where $\lambda$ is the wavelength of the observation, $d_{\mathrm{M}}$ and $d_{\mathrm{OM}}=D_{\mathrm{os}}-d_{\mathrm{M}}$ are the distance to the Moon from the Earth and from the source star, and $r=d_{\mathrm{M}} \theta$. Since $d_{\mathrm{OM}} \gg d_{\mathrm{M}}$, one can approximate eq. (3.1.3) by $z=r\left(2 / \lambda d_{\mathrm{M}}\right)^{1 / 2}$. When the image of a star is directly on the edge of the Moon's surface, $v=r=0, \mathcal{C}(0)=\mathcal{S}(0)=0$ and $g / g_{0}=0.25$.

\subsection{Simulation}

In our simulation the observations are assumed to be carried out as follows. During an occultation event, photometry is carried out continuously (i.e., with time resolution $\ll 1$ mas) and read instantaneously. This kind of high speed photometry technique has already been developed and the actual instrument (HSP) had been installed at the Hubble Space Telescope (HST) although the instrument was removed from HST to accommodate the installation of corrective optics. A large $(\gtrsim 4 \mathrm{~m})$ telescope is required for the measurement to compensate for the relative faintness of even giant sources in the bulge. For an $H=0$ star, a (single channel) photometer can detect $\sim 9.4 \times 10^{9} \eta \Delta \lambda$ photons $\mathrm{m}^{-2} \mathrm{~s}^{-1} \mu \mathrm{m}^{-1}$. With 
a band width of $\Delta \lambda=0.3 \mu \mathrm{m}$ and assuming a detection efficiency $\eta=0.5$, it can detect $1.6 \times 10^{7}$ photons $\mathrm{ms}^{-1}$ using a $4 \mathrm{~m}$ telescope with $90 \%$ effective surface area. The expected number of photons from a bulge clump giant, typically $H=13.2 \mathrm{mag}$ (Tiede, Frogel, \& Terndrup 1995), will be $\sim 67 \mathrm{~ms}^{-1}$ assuming an extinction of $A_{H}=0.25$ mag toward the bulge. The sky has a brightness of $14 \mathrm{mag}_{\operatorname{arcsec}^{-2}}$ in the $H$ band producing an expected sky flux of 71 photons $\mathrm{ms}^{-1}$ in an aperture of 1.5 arcsec in diameter. We assume that the occultation occurs when the dimensionless impact parameter $x=0.5$, and thus the magnification $A=2.18$. Then the individual magnifications of the image are

$$
A_{ \pm}=\frac{x_{ \pm}^{2}}{x_{+}^{2}-x_{-}^{2}} ; \quad x_{ \pm}=\frac{\sqrt{x^{2}+4} \pm x}{2}
$$

giving $A_{+}=1.59$ and $A_{-}=0.59$ for the primary and secondary images, respectively. The photon counts for each image are then $N_{\nu, 1}=112$ photons $\mathrm{ms}^{-1}$ and $N_{\nu, 2}=41$ photons $\mathrm{ms}^{-1}$. The expected number of detected photons from the individual images and from the sky for various observational situations are computed and listed in Table 1. In the table the signal-to-noise ratio is computed by $S / N=N_{\nu} /\left(N_{\nu, 1}+N_{\nu, 2}\right)^{1 / 2}$. We assume that other sources of noise, e.g., dark current and read-out noise, are negligible.

The theoretically-expected fringe pattern $g / g_{0}$ in the $H$ band, centered at $1.65 \mu \mathrm{m}$, is computed by eq. (3.1.1) and is shown as a function time $t$ in Figure 1 (a). In the figure, time is measured from the moment when the first (closer to the Moon) image just crosses the Moon's limb and negative time implies that the image is behind the Moon. The angular distance is related to time scale by

$$
\theta=\omega t \cos \psi
$$


where $\omega$ is the orbital angular speed of the Moon and $\psi$ is the angle of the lunar limb relative to the direction of the Moon's motion. The total number of photons in the signal is normalized into unity when $t=\infty$. Due to the phase shift of the Fresnel integrals with time, there are beat patterns. In the simulation, we assume the angular separation between the two images of lensed stars is $\phi_{\text {sep }}=3$ mas, equivalent to an Einstein radius $\theta_{e} \sim 1.5$ mas, which would be typical for a disk lens located at $\sim 2 \mathrm{kpc}$ with mass of $M=0.7 M_{\odot}$. Since the long events are probably caused by relatively massive MACHOs, the expected separation could be greater.

Up until now, our computation has been based on a monochromatic wave observation. However, the $H$-band filter has a finite bandpass. For the correction of band width in our analysis, we average the flux weighted by the filter function. The filter function in the $H$ band is well-approximated by the tophat function $\omega_{\text {filter }}:$

$$
w_{\text {filter }}= \begin{cases}\omega_{0}, & 1.5 \mu \mathrm{m} \leq \lambda \leq 1.8 \mu \mathrm{m} \\ 0, & \text { otherwise }\end{cases}
$$

where $\omega_{0}=(0.3 \mu \mathrm{m})^{-1}$. The resultant fringe pattern $h(\theta)$, after being averaged by the filter function, is shown in Figure 1 (b). The small-scale fluctuation in flux is smeared out, and thus the beat patterns, which might have been useful for the measurement of $\phi_{\text {sep }}$, disappear.

We make the simulation more realistic by including the beam pattern of the telescope. At any time, one edge of the telescope mirror will see an image displaced by a small angle compared to the image seen at the center of the mirror. The resulting image is then the combination of all images seen by different parts of the 
mirror. The beam pattern decreases with increasing angular separation $\theta_{\mathrm{p}}$ from the center of the mirror because the relative area of the mirror in a strip located at $\theta_{\mathrm{p}}$ decreases as $\theta_{\mathrm{p}}$ increases. For a circular mirror $f_{\text {beam }}=\left[1-\left(\theta_{\mathrm{p}} / \theta_{\text {tel }}\right)^{2}\right]^{1 / 2}$, where $\theta_{\text {tel }}=a / 2 d_{\mathrm{M}}=1$ mas for an assumed aperture $a=4 \mathrm{~m}$. Then the expected intensity $h(\theta)$ is obtained by convolving the intensity $h(\theta)$ with the beam pattern $f_{\text {beam }}\left(\theta_{\mathrm{p}}\right)$ :

$$
I(\theta)=\int f_{\text {beam }}\left(\theta_{\mathrm{p}}-\theta\right) h\left(\theta_{\mathrm{p}}\right) d \theta_{\mathrm{p}}
$$

The resulting fringe pattern after the beam pattern convolution and being averaged by filter function is shown in Figure 1 (c).

\section{Uncertainty of Measurement and Observational Strategy}

The determination of the angular separation of the two images of a lensed star is made by fitting the observed fringe pattern to a set of light curves with different $\phi_{\text {sep }}$. This fitting process suffers from another uncertainty in addition to the one from the angular separation of the images. This uncertainty comes from the ambiguity of a reference point, which is the time of first image occultation, (i.e., $\theta=0)$. A misalignment of the reference point would result in misinterpretation of $\phi_{\text {sep }}$. Therefore, we include the misalignment quantified by the shift in the reference point, $\Delta t_{0}$, as a free parameter in our analysis.

The contours of equal uncertainties measured by $\chi^{2}$ in the parameter space of $\Delta t_{0}$ and $\phi_{\text {sep }}$ are shown in Figure 2 (a) for the first lensing event described in Table 1. The contour levels are drawn at $1 \sigma, 2 \sigma$, and $3 \sigma$ levels from the point of minimum $\chi^{2}$ marked by " $\mathrm{x}$ ". In the contour map there appear two minima which 
are located symmetrically about $\phi_{\text {sep }}=0$. These two minima appear because of the ambiguity of order of occultation: the fringe pattern when the primary image approaches the Moon first looks similar to the pattern when the other image approaches first. If the two images have exactly the same intensity, there will be no difference in the resultant fringe patterns. The uncertainty is, $\Delta \phi_{\text {sep }} \sim 0.55$ mas at the $1 \sigma$ level. This would be a significant detection although not as precise as one might like.

However, the uncertainty decreases significantly with increasing $S / N$ ratio. There are two ways to increase the $S / N$ ratio; observing bright stars or highlymagnified events. For illustration, we compute the uncertainties for events under exactly the same conditions as the previous case except for a higher magnification $A=3.0$ [Fig. 2 (b)] and a 0.5 mag brighter source star [Fig. 2 (c)], which are the second and third cases in Table 1, respectively. For both cases the uncertainty is $\Delta \phi_{\text {sep }} \sim 0.35$ mas at the $1 \sigma$ level.

\section{Practical Considerations}

\subsection{Lunar Topography}

For an actual observation, one is required to consider some miscellaneous factors that make $\phi_{\text {sep }}$ deviate somewhat from what we have assumed in the computation. The first deviation arises because the Moon's limb is not a perfect straight line but has a small curvature. If the angular separation between images is big enough (e.g., binary stars), the observed pattern would slightly deviate from the Fresnel diffraction pattern, which assumes an infinite straight-line surface. How- 
ever, for the scale of $\lesssim 5.5 \mathrm{~m}$, which is equivalent to $\phi_{\text {sep }} \lesssim 3$ mas projected on the Moon's surface, the Moon's limb is a nearly perfect straight line. Indeed, typical errors arising from lunar surface curvature are negligible even for the measurement of a few tens of arcsec (e.g., Richichi et al. 1994). The other type of deviation which does affect on the measurement of $\phi_{\text {sep }}$ is due to topographical features on the lunar surface. If the images are occulted at different parts of a geological structure on the Moon, e.g., moutains and valleys, the angle $\psi$ in eq. (3.2.2) deviates from what one assumes based on a smooth circular lunar disk. Assuming that the deviation due to large scale strucures of order $\mathrm{km}$ or larger can be corrected using a detailed lunar map, one still has a problem due to smaller-scale structures such as rocks and cliffs. Fortunately, lunar craters and other topographical features are generally not very rugged, and even mountains are very smooth (Abell, Morrison, \& Wolff 1993).

\subsection{Orientation of Images}

We have assumed up until now that the orientation of the two images is perpendicular to the approaching limb of the Moon. However, the orientation in general is random and thus what one measures is the component of $\phi_{\text {sep }}$ normal to the Moon's limb not the intrinsic separation of interest. If one knows exactly how the source star crosses the Einstein ring, it is simple geometry to deduce $\phi_{\text {sep }}$ from the measured angular separation $\phi_{i}$, where the subscript " $i$ " is explained below. However, resolving the orientations of the images is not a trivial problem and requires additional information.

Information about the lens geometry can be obtained from (ground-based) 
measurement of the parallax. At a minimum, parallax measurements determine the component of the projected velocity parallel to the ecliptic and the magnitude of the component normal to the ecliptic. In general, this still leaves a four-fold degeneracy: two-fold for the sign of the normal component and two-fold for the sense of source motion (clockwise or counterclockwise) relative to observer-lens line of sight. In some cases, especially, if an event is observed away from the ecliptic and has a long time scale, this four-fold degeneracy is completely broken by the parallax measurement itself. Indeed, this is the case for the long-event parallax measured by Bennett et al. (1995). In these cases the geometry is unambiguous and $\theta_{e}$ can be determined directly from $\phi_{\text {sep. }}$. However, it is difficult to break this degeneracy when the event is close to the ecliptic. Since the Moon never gets farther than $\sim 5^{\circ}$ from the ecliptic, it is also important to consider the degenerate case.

The four-fold degeneracy can be broken by carrying out occultation observations twice so that the approaching angle, $\theta_{\mathrm{A}}$, of the Moon's limb is different for each observation. This can be done either by observing the occultation at two different locations on the Earth or by observing an occultation from the same location a month later. The latter case is possible for long events since the declination of Moon's orbit typically changes by $\sim 1 / 3$ of its diameter per month at fixed right ascension. The four-fold degeneracy of lens geometries is illustrated in Figure 4: transverse velocities pointing below and above the ecilptic are noted by "case I" and "case II". Subnotations, "(a)" and "(b)", describe the clockwise and counterclockwise source crossings. For illustration, we assume that the Moon's limb is approaching the images with $\theta_{\mathrm{A}}=45^{\circ}$ ("/" sense) and $\theta_{\mathrm{A}}=-45^{\circ}$ (" " sense). 
For each observation shown in the figure the shaded surface indicates the orientation of the surface of the Moon and the shaded arrow indicates the direction of motion. The angular separation that is measured when $\theta_{\mathrm{A}}=45^{\circ}$ and $\theta_{\mathrm{A}}=-45^{\circ}$ is marked by $\phi_{1}$ and $\phi_{2}$. For each case the ratios between measured angular separations determined at two different locations (or times), $\phi_{1} / \phi_{2}$, are different from one another. Therefore, one can obtain the full lens geometry by ruling out the other three cases from a comparison of the observed ratio $\phi_{1} / \phi_{2}$ with the expected ratios.

\subsection{Event Rate}

One can measure proper motions using lunar occultations for about one long time-scale lensing event per year. The occulation can be observed in a strip of sky along the path of the Moon with a width larger than the angular size of the Moon by observing the occultation at higher (or lower) Earth latitudes, $\phi$. The effective occulting cross-section is $\sim 1^{\circ} .5$ assuming the observations could be arranged at places within $\phi= \pm 30^{\circ}$. Then the area of sky toward the bulge, with width $\pm 5^{\circ}$ around the Galactic plane, swept by the effective cross-section is $15 \mathrm{deg}^{2}$. If the observation could be carried out at more extreme Earth latitudes, the event rate could be increased. The MACHO group has detected 13 giant events out of a total of 44 events during a bulge season by covering $12 \mathrm{deg}^{2}$ of sky. Three of 44 are long events. The expected occultation event rate is then $3 \times(13 / 44) \times(15 / 12) \sim 1$ event/yr. This is substantially higher than the number of proper motions of long lensing events that can be obtained using other methods. Another advantage of the occultation method is that for the long events the measurement could be repeated 
for better determination of proper motion. Special attention should be paid to observing those fields which the Moon will occult sometime during a given bulge season. For some regions close to the plane, optical observations are impossible due to extinction. However, events can still be detected using $H$ or $K$ band observations. Indeed, the long events may well be concentrated near the plane (see Fig. 1 from Bennett et al. 1995). Because the long events last many months, it would be sufficient to make these infrared observations once per week compared to once per day for standard optical observations.

Acknowledgement: We would like to thank M. Everett and G. Newsom for making very helpful comments. 


\section{REFERENCES}

1. Abell, G. O., Morrison, D., Wolff, D. C. 1993, Exploration of the Universe (Asunder College Publishing, Philadelphia), 222

2. Alcock, C. et al. 1995, ApJ, 445, 133

3. Bennett, D. P. et al. 1995, Proceedings of the Fifth Annual Maryland Meeting on Astrophysics: Dark Matter, S. S. Holt, C. L. Bennett, eds., in press

4. Gould, A. 1992, ApJ, 392,442

5. - 1994, ApJ, 421, L71

6. -1995, ApJ, 447, 000

7. Gould, A., Miralda-Escudé, J., \& Bahcall, J. N. 1994, ApJ, 423, L105

8. Griest, K. et al. 1991, ApJ, 387, 181

9. Han, C., \& Gould, A. 1995a, ApJ, 447, 000

10. 1 1995b, ApJ, submitted

11. Hecht, E. \& Zajac, A. 1979, Optics (Addison-Wesley Publishing Company, Reading), 385

12. Kiraga, M., \& Paczyński, B. 1994, ApJ, 430, L101

13. Nemiroff, R. J. \& Wickramasinghe, W. A. D. T. 1994, ApJ, 424, L21

14. Richichi, A., Calamai, G., \& Leinert, C. 1994, A\&A, 286, 829

15. Tiede, G. P., Frogel, J. A., \& Terndrup, D. M. 1995, AJ, submitted

16. Udalski, A., Szymański, M., Stanek, K. Z., Kalużny, J., Kubiak, M., Mateo, M., Krzemiński, B., \& Venkat, R. 1994, Acta Astron., 44, 165 
17. Witt, H. 1995, ApJ, in press

18. Zhao, H., Spergel, D. N., \& Rich, R. M. 1995, ApJ, 440, L13 